\title{
Open Access Series of Imaging Studies: Longitudinal MRI Data in Nondemented and Demented Older Adults
}

\section{Citation}

Marcus, Daniel S., Anthony F. Fotenos, John G. Csernansky, John C. Morris, and Randy L. Buckner. 2010. "Open Access Series of Imaging Studies: Longitudinal MRI Data in Nondemented and Demented Older Adults." Journal of Cognitive Neuroscience 22 (12). MIT Press - Journals: 2677-84. doi:10.1162/jocn.2009.21407.

\section{Permanent link}

http://nrs.harvard.edu/urn-3:HUL.InstRepos:41555750

\section{Terms of Use}

This article was downloaded from Harvard University's DASH repository, and is made available under the terms and conditions applicable to Other Posted Material, as set forth at http:// nrs.harvard.edu/urn-3:HUL.InstRepos:dash.current.terms-of-use\#LAA

\section{Share Your Story}

The Harvard community has made this article openly available.

Please share how this access benefits you. Submit a story.

\section{Accessibility}




\title{
Open Access Series of Imaging Studies: Longitudinal MRI Data in Nondemented and Demented Older Adults
}

\author{
Daniel S. Marcus ${ }^{1}$, Anthony F. Fotenos', John G. Csernansky ${ }^{2}$, \\ John C. Morris ${ }^{1}$, and Randy L. Buckner ${ }^{3,4,5,6}$
}

\begin{abstract}
The Open Access Series of Imaging Studies is a series of neuroimaging data sets that are publicly available for study and analysis. The present MRI data set consists of a longitudinal collection of 150 subjects aged 60 to 96 years all acquired on the same scanner using identical sequences. Each subject was scanned on two or more visits, separated by at least 1 year for a total of 373 imaging sessions. Subjects were characterized using the Clinical Dementia Rating (CDR) as either nondemented or with very mild to mild Alzheimer's disease. Seventy-two of the subjects were characterized as nondemented throughout the study. Sixty-four of the included subjects were characterized as demented at the time of their initial visits and remained so for subsequent scans, including 51 individuals with CDR 0.5 similar level of impairment
\end{abstract}

\section{INTRODUCTION}

The Open Access Series of Imaging Studies (OASIS) is a project aimed at making neuroimaging data sets of the brain freely available to the scientific community. By compiling and freely distributing neuroimaging data sets, we hope to facilitate future discoveries in basic and clinical neuroscience similar to other initiatives such as the Alzheimer's Disease Neuroimaging Initiative. The initial OASIS set of cross-sectional MRI data included over 400 demented and nondemented individuals across the adult lifespan (Marcus, Wang, et al., 2007). Here we describe a longitudinal sample of MRI data from older adults, with and without Alzheimer's disease (AD). The preparation and release of the data set follows the rigor established with the initial release: careful quality control, detailed documentation, example of postprocessed images, full anonymization, multiple access methods, ongoing support, and liberal data usage requirements (Marcus, Wang, et al., 2007).

The data set includes longitudinal MRI data from 150 individuals age 60 to 96 years, including 64 individuals with very mild to moderate $\mathrm{AD}$ as diagnosed clinically and characterized using the Clinical Dementia Rating (CDR) scale (Morris et al., 2001; Morris, 1993) at their initial visit. Another

\footnotetext{
${ }^{1}$ Washington University School of Medicine, ${ }^{2}$ Northwestern University School of Medicine, ${ }^{3}$ Harvard University, ${ }^{4}$ Harvard Medical School, ${ }^{5}$ Massachusetts General Hospital, ${ }^{6}$ Howard Hughes Medical Institute
}

to individuals elsewhere considered to have "mild cognitive impairment." Another 14 subjects were characterized as nondemented at the time of their initial visit (CDR 0) and were subsequently characterized as demented at a later visit $(\mathrm{CDR}>0)$. The subjects were all right-handed and include both men $(n=62)$ and women $(n=88)$. For each scanning session, three or four individual T1-weighted MRI scans were obtained. Multiple withinsession acquisitions provide extremely high contrast to noise, making the data amenable to a wide range of analytic approaches including automated computational analysis. Automated calculation of whole-brain volume is presented to demonstrate use of the data for measuring differences associated with normal aging and Alzheimer's disease.

14 of the individuals were characterized as nondemented at the time of one or more scans and then clinically determined to have $\mathrm{AD}$ at the time of a subsequent scan. All data were acquired on the same scanner using identical procedures. Subjects were screened to eliminate individuals with psychiatric and neurological conditions that might contribute to dementia but, where possible, variation typical of advanced aging was included. Thus, many of the older adults had age-related increases in blood pressure and a small percentage treated diabetes. Sample characteristics were similar between the individuals with and without $\mathrm{AD}$.

Longitudinal brain imaging has proven useful in studying normal and diseased aging. Whole-brain volume decline as measured from longitudinally acquired MRI has been shown to evolve at a near $0.5 \%$ per year (in the range -0.37 to -0.88 across seven reports; Fotenos, Snyder, Girton, Morris, \& Buckner, 2005; Jack et al., 2004; Liu et al., 2003; Resnick, Pham, Kraut, Zonderman, \& Davatzikos, 2003; Thompson et al., 2003; Wang \& Doddrell, 2002; Chan et al., 2001) in nondemented older adults, somewhat greater than that observed in younger adults (Raz et al., 2005; Liu et al., 2003). The volumes of the whole-brain and structures associated with memory have been shown to atrophy at a significantly greater rate in mild cognitive impairment and early AD (e.g., Dickerson et al., 2006; Fotenos et al., 2005; Killiany et al., 2000, 2002; Fox \& Freeborough, 1997; Jack et al., 1997; Jack, Petersen, O’Brien, \& Tangalos, 
1992). Longitudinal analysis of measures derived from advanced computational methods, including nonlinear deformations and shape analysis, have also revealed ageand disease-associated changes in the brain (e.g., Buckner et al., 2005; Thompson et al., 2003; Wang et al., 2003; Scahill, Schott, Stevens, Rossor, \& Fox, 2002). Longitudinal measures of brain structure are emerging as tools for tracking progression of disease and as adjunct outcome measures in clinical trials (e.g., Jack et al., in press; Borroni, Premi, Di Luca, \& Padovani, 2007; Chong, Lim, \& Sahadevan, 2006; Mueller et al., 2005). The OASIS longitudinal data set is being made openly available to encourage continued investigation into aging and disease processes and to support development of improved methods for studying these processes.

\section{METHODS}

\section{Subjects}

Subjects aged 60 to 96 years were selected from a larger database of individuals who had participated in MRI studies at Washington University on the basis of the availability of at least two separate visits in which clinical and MRI data were obtained, at least three acquired T1-weighted images per imaging session, and right-hand dominance. Subjects were obtained from the longitudinal pool of the Washington University Alzheimer Disease Research Center (ADRC). The ADRC's normal and cognitively impaired subjects were recruited primarily through media appeals and word of mouth, with $80 \%$ of subjects initiating contact with the center and the remainder being referred by physicians. All subjects participated in accordance with guidelines of the Washington University Human Studies Committee. Approval for public sharing of the anonymized data was also specifically obtained.

All subjects were screened for inclusion in this release. Each subject underwent the ADRC's full clinical assessment as described below. Subjects with a primary cause of dementia other than $\mathrm{AD}$ (e.g., vascular dementia, primary progressive aphasia), active neurologic or psychiatric illness (e.g., major depression), serious head injury, history of clinically meaningful stroke, and use of psychoactive drugs were excluded, as were subjects with gross anatomical abnormalities evident in their MRI images (e.g., large lesions, tumors). However, subjects with age-typical brain changes (e.g., mild atrophy, leukoaraiosis) were accepted. MRI acquisitions typically were obtained within 1 year before or after a subject's clinical assessment (mean $=111$ days, range $=0-352$ days). Twelve subjects with $\mathrm{AD}$ were scanned after a somewhat longer duration ( mean $=653$ days, range $=$ 374-924 days) but were included because each had several previous clinical assessments with CDR scores greater than 0 . Two subjects without dementia were scanned somewhat longer than 1 year before a clinical assessment $(392$ and 431 days) but were included because their subsequent clinical assessments continued to indicate no signs of de- mentia. Each subject was scanned on two or more separate occasions, with an average delay of 719 days (range $=$ 183-1707 days) between visits. The final data set includes 150 subjects and 373 imaging sessions.

Portions of the clinical, demographic, and longitudinal image data obtained from subjects in this release have been used in previous publications (Dickerson et al., 2008; Fotenos, Mintun, Snyder, Morris, \& Buckner, 2008; He, Chen, \& Evans, 2008; Salat et al., 2009; Dickerson et al., 2009; Buckner et al., 2005; Burns et al., 2005; Fotenos et al., 2005; Head, Snyder, Girton, Morris, \& Buckner, 2005; Buckner et al., 2004; Salat et al., 2004). Many of the subjects were part of the cross-sectional OASIS data set (Marcus, Olsen, et al., 2007; Marcus, Wang, et al., 2007) but have been assigned new random identifiers.

\section{Clinical Assessment}

Dementia status was established and staged using the CDR scale. The determination of $\mathrm{AD}$ or nondemented control status is based solely on clinical methods, without reference to psychometric performance, and any potential alternative causes of dementia (known neurological, medical, or psychiatric disorders) must not contribute to dementia. The diagnosis of $\mathrm{AD}$ is based on clinical information (derived primarily from a collateral source) that the subject has experienced gradual onset and progression of decline in memory and other cognitive and functional domains. Specifically, the CDR is a dementia-staging instrument that rates subjects for impairment in each of six domains: memory, orientation, judgment and problem solving, function in community affairs, home and hobbies, and personal care. On the basis of the collateral source and subject interview, a global CDR score is derived from individual ratings in each domain. A global CDR of 0 indicates no dementia and a CDR of $0.5,1,2$, and 3 represent very mild, mild, moderate, and severe dementia, respectively. These methods allow for the clinical diagnosis of $\mathrm{AD}$ in individuals with a CDR of 0.5 or greater on the basis of standard criteria that is confirmed by histopathological examination in $93 \%$ of the individuals (Berg et al., 1998), even for those in the earliest symptomatic stage (CDR 0.5) of $\mathrm{AD}$ who elsewhere may be considered to represent "mild cognitive impairment" (Storandt, Grant, Miller, \& Morris, 2006).

\section{Image Acquisition}

For each subject, three to four individual T1-weighted magnetization prepared rapid gradient-echo (MP-RAGE) images were acquired on a 1.5-T Vision scanner (Siemens, Erlangen, Germany) in a single imaging session. Head movement was minimized by cushioning and by a thermoplastic face mask. Headphones were provided for communication. A vitamin E capsule was placed over the left forehead to provide a reference marker of anatomic side. Positioning was low in the head coil (toward the feet) to 
optimize imaging of the cerebral cortex. MP-RAGE parameters were empirically optimized for gray-white contrast (Table 1). The scanner and the sequences were maintained across the duration of the study so the present data are not influenced by hardware upgrades or other instrument differences.

\section{Postprocessing}

For each subject, the individual scan files were converted from Siemens proprietary IMA format into 16-bit NiFTI1 format using a custom conversion program. Header fields with identifying information (patient ID, experiment date) were left blank. The images were then corrected for interscan head movement and spatially warped into the atlas space of Talairach and Tournoux (1988) using a rigid transformation that differs in process from the original piecewise scaling. The resulting transformation nonetheless places the brains in the same coordinate system and bounding box as the original atlas. The template atlas used here consisted of a combined young-and-old target previously generated from a representative sample of young $(n=12)$ and nondemented old $(n=12)$ adults. The use of a combined template has been shown to minimize the potential bias of an atlas normalization procedure to overexpand atrophied brains (Buckner et al., 2004). Given the age range of the present sample, an old-only atlas target could have been used. We chose to retain the young-and-old target to be comparable to our earlier report (Marcus, Wang, et al., 2007).

For registration, a 12-parameter affine transformation was computed to minimize the variance between the first MP-RAGE image and the atlas target. The remaining MP-RAGE images were registered to the first (in-plane stretch allowed) and resampled via transform composition into a 1-mm isotropic image in atlas space. The result was a single, high-contrast, averaged MP-RAGE image in atlas space. Subsequent steps included skull removal by application of a loose-fitting atlas mask and correction for intensity inhomogeneity because of nonuniformity in the magnetic

Table 1. MR Image Acquisition Details

\begin{tabular}{ll}
\hline Sequence & MP-RAGE \\
TR (msec) & 9.7 \\
TE (msec) & 4.0 \\
Flip angle & $10^{\circ}$ \\
TI (msec) & 20 \\
TD (msec) & 200 \\
Orientation & Sagittal \\
Thickness, gap (mm) & $1.25,0$ \\
Slice number & 128 \\
Resolution & $256 \times 256(1 \times 1 \mathrm{~mm})$ \\
\hline
\end{tabular}

field. Intensity variation was corrected across contiguous regions on the basis of a quadratic inhomogeneity model fitted to data from a phantom.

\section{Estimated Total Intracranial Volume and Normalized Whole-brain Volume}

The procedures used for measuring intracranial and wholebrain volumes have been described previously (Fotenos et al., 2005; Buckner et al., 2004) and are identical to our earlier OASIS data release (Marcus, Wang, et al., 2007). Estimated total intracranial volume (e'TIV) was computed by scaling the manually measured intracranial volume of the atlas by the determinant of the affine transform connecting each individual's brain to the atlas. This method is minimally influenced and proportional to manually measured total intracranial volume.

Normalized Whole-brain Volume (nWBV) was computed using the FAST program in the FSL software suite (www. fmrib.ox.ac.uk/fsl). The image was first segmented to classify brain tissue as cerebral spinal fluid, gray, or white matter. The segmentation procedure iteratively assigned voxels to tissue classes on the basis of maximum likelihood estimates of a hidden Markov random field model. The model used spatial proximity to constrain the probability with which voxels of a given intensity are assigned to each tissue class. Finally, nWBV was computed as the proportion of all voxels within the brain mask classified as tissue (either gray or white matter). The unit of normalized volume is percent, which represents the percentage of the total white and gray matter voxels within the eTIV (Fotenos et al., 2005). To calculate atrophy rates, we estimated the slope of the line connecting nWBV measurements within each individual, divided by baseline nWBV, expressed as percent change per year. For example, in a participant with two scans, atrophy rate was computed as nWBV at Scan 2 minus nWBV at Scan 1, divided by the interval between measurements, divided by nWBV at Scan 1, times 100. ANCOVA was again used to test for differences in atrophy rate on the basis of age, sex, and dementia status.

\section{Quality Control}

All images in the data set were carefully screened for artifacts, acquisition problems, and processing errors. During the screening process, each image was viewed on a per-slice basis along the axis of acquisition. Typical flaws visible in the images included electronic noise resulting in bright lines through multiple slices, motion artifacts appearing as hazy bands across the image, poor head positioning resulting in wraparound artifacts, distortions from dental work, and limited image contrast. Images with severe flaws were excluded from the data set. A number of borderline images remain in the distribution, providing tool builders and testers with a realistic range of acquisition quality. In cases where individual scans were deemed unusable, the single scan was removed from the data set but the remainder 
Table 2. Age and Diagnosis Characteristics of Subjects at the Time of Their Initial Visit

\begin{tabular}{|c|c|c|c|c|c|c|c|c|c|c|c|}
\hline \multirow[b]{2}{*}{ Age Group } & \multirow[b]{2}{*}{$N$} & \multicolumn{5}{|c|}{ Nondemented } & \multicolumn{5}{|c|}{ Demented } \\
\hline & & $n$ & Mean & Male & Female & Convert & $n$ & Mean & Male & Female & CDR 0.5/1 \\
\hline $60 \mathrm{~s}$ & 34 & 23 & 65.71 & 6 & 17 & 3 & 11 & 65.67 & 8 & 3 & $8 / 3$ \\
\hline $70 \mathrm{~s}$ & 71 & 35 & 74.91 & 11 & 24 & 4 & 36 & 73.97 & 20 & 16 & $29 / 7$ \\
\hline $80 \mathrm{~s}$ & 41 & 26 & 84.30 & 9 & 17 & 7 & 15 & 82.33 & 7 & 8 & $13 / 2$ \\
\hline $90 \mathrm{~s}$ & 4 & 2 & 92.50 & 0 & 2 & 0 & 2 & 93.00 & 1 & 1 & $1 / 1$ \\
\hline Total & 150 & 86 & 75.82 & 26 & 59 & 14 & 64 & 74.95 & 36 & 29 & $52 / 13$ \\
\hline
\end{tabular}

The Convert column indicates individuals who were determined to have $\mathrm{AD}$ on a subsequent visit. CDR $=$ Clinical Dementia Rating, with $0,0.5$, and 1 corresponding to nondemented, very mild, and $\mathrm{AD}$, respectively. CDR 0.5 individuals may be considered to represent those elsewhere labeled as "mild cognitive impairment" (MCI).

of the subject's scans was included. Overall, 25 imaging sessions were excluded from the final release because of poor image quality.

\section{RESULTS}

\section{Overview of the Data Set}

The current data set consists of 150 subjects (88 women) aged 60 to 96 years (Table 2). At the time of their initial visit, 86 had a CDR score of 0 , indicating no dementia, and 64 had a CDR score greater than 0 (52 subjects, $C D R=0.5$; 13 subjects, $\mathrm{CDR}=1$; 0 subject, $\mathrm{CDR}=2$ ), indicating a diagnosis of very mild to moderate $\mathrm{AD}$. Of the subjects who were initially determined to be nondemented, 14 were later determined to be demented (CDR $>0)$ at the time of a subsequent imaging visit. Additional demographics and clinical characteristics of the subjects are shown in Table 3.

\section{Anatomic Characteristics}

Whole-brain volumes for each of the subjects in the data set are plotted by age at each visit in Figure 1. Marked decreases in nWBV are apparent with age, and nWBV is significantly impacted by dementia status (Figure 2). Differences in nWBV between nondemented (CDR 0), very mild dementia (CDR 0.5), and mild dementia (CDR 1) are all significant $(p<.01)$. Whole-brain atrophy rates are shown in Figure 3 (top). The atrophy rate in nondemented individuals was $-0.49 \%(S D=0.56)$ per year. The atrophy rate in individuals with DAT was $-0.87 \%(S D=0.99)$ per year, a significant increase compared with nondemented individuals $(p<.01)$. For the 14 individuals who declined from an initial CDR 0 to a CDR 0.5 at the time of their last scan, the atrophy rate fell between nondemented individuals and those who entered with $\mathrm{AD}(-0.69 \%$ per year, $S D=0.62)$.

Table 3. Sample Characteristics of Subjects

\begin{tabular}{lccc}
\hline & CDR 0 & CDR 0.5 & CDR 1 \\
\hline Number & 86 & 51 & 13 \\
Female/male & $60 / 26$ & $21 / 30$ & $7 / 6$ \\
Age (years) & $75.8 \pm 8.2(60-93)$ & $74.8 \pm 6.3(62-90)$ & $75.7 \pm 8.7(61-96)$ \\
Education (years) & $15.2 \pm 2.7(8-23)$ & $13.6 \pm 2.8(6-20)$ & $14.0 \pm 3.2(8-20)$ \\
MMSE & $29.1 \pm 0.8(27-30)$ & $26 \pm 3.1(17-30)$ & $23.0 \pm 3.3(19-30)$ \\
Prescriptions (n) & $2.9 \pm 2.1(0-9)$ & $143.5 \pm 19.4(118-188)$ & $2.5 \pm 2.4(0-7)$ \\
Systolic BP (mmHg) & $135.5 \pm 20.3(98-192)$ & $77.1 \pm 10.1(58-98)$ & $143.4 \pm 24.9(90-188)$ \\
Diastolic BP (mmHg) & $72.8 \pm 10.2(50-100)$ & 46.0 & $76.9 \pm 9.2(60-88)$ \\
Reported HBP (\%) & 54.6 & 14.0 & 53.3 \\
Diabetes (\%) & 9.3 & 13.3 \\
\hline
\end{tabular}

The sample consisted of 150 individuals ( 72 nondemented, 64 with $\mathrm{AD}$, and 14 who converted over the course of the study). Clinical measures in the above table were obtained at the clinical assessment closest in date to the initial imaging session, except in 39 cases where these values were not available until a later visit. Values are presented as mean $\pm S D$. Values in parentheses represent the range. Compared with the nondemented adults, the older adults with dementia had lower scores on the MMSE $(p<.001)$ and slightly fewer years of education $(p<.001)$.

$\mathrm{CDR}=$ Clinical Dementia Rating, with 0, 0.5, and 1 corresponding to nondemented, very mild, and mild AD, respectively; MMSE = Mini-Mental State Examination where scores range from 30 (best) to 0 (worst); HBP $=$ high blood pressure. 


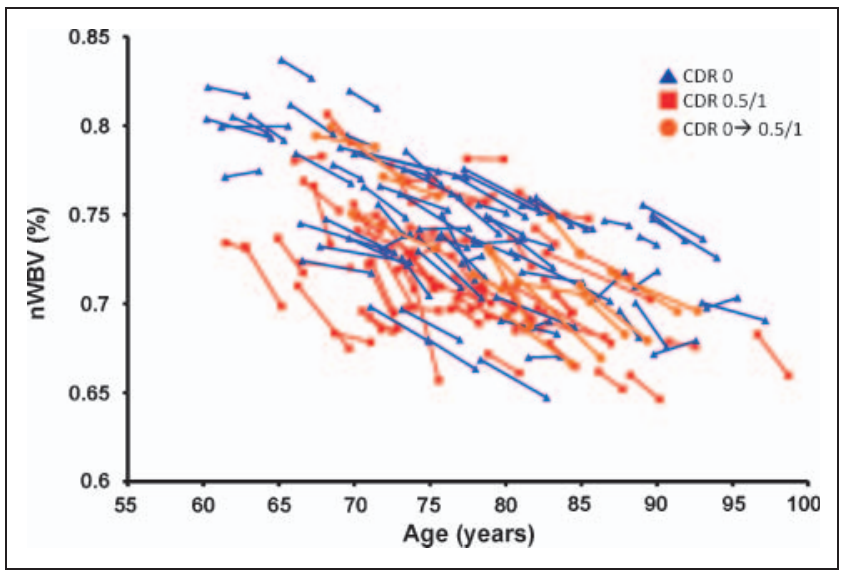

Figure 1. Longitudinal plot of $n W B V$; lines connect $n W B V$ at baseline and follow-up scans (or the best fit, for participants with multiple follow-ups), such that the slope of each line as a proportion of baseline nWBV represents an individual's atrophy rate.

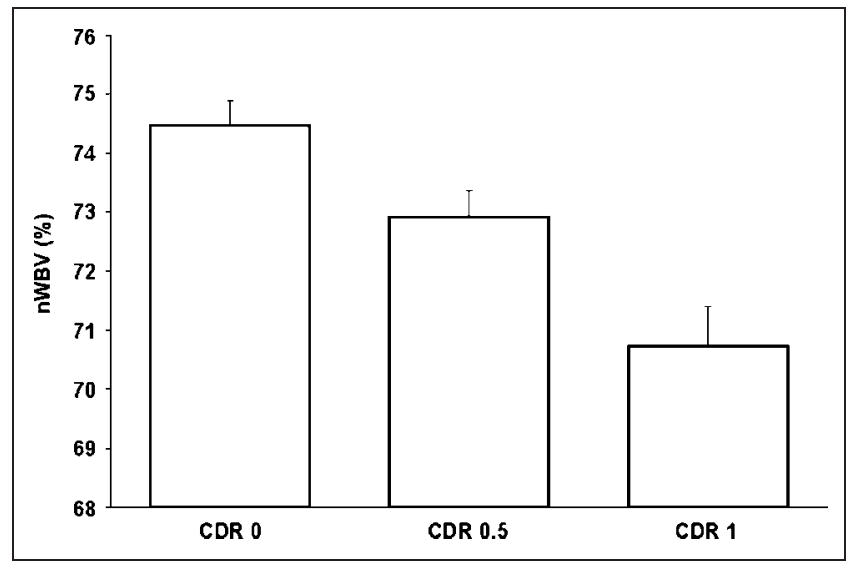

Figure 2. Mean cross-sectional nWBV for all individuals separated by Clinical Dementia Rating (CDR) $(0,0.5$, and 1$)$ at the individuals' initial visit. All differences are significant.
Figure 3. (A) Longitudinal atrophy rates, expressed in nWBV loss per year relative to baseline, are separated by CDR status at first and last session. Atrophy rate was significantly greater for the group entering the experiment with very mild dementia (CDR $0.5 \rightarrow 0.5 / 1$ ) than the group entering without dementia that remained stable $(\mathrm{CDR} 0 \rightarrow 0$ ), whereas the rate for the group that manifested the earliest signs of $\mathrm{AD}$ during the experiment $(\mathrm{CDR} 0 \rightarrow 0.5)$ fell between those with no dementia and those who entered with dementia.

(B) Individual atrophy rates plotted by age and CDR score. The trendline is plotted for CDR $0 \rightarrow 0$.

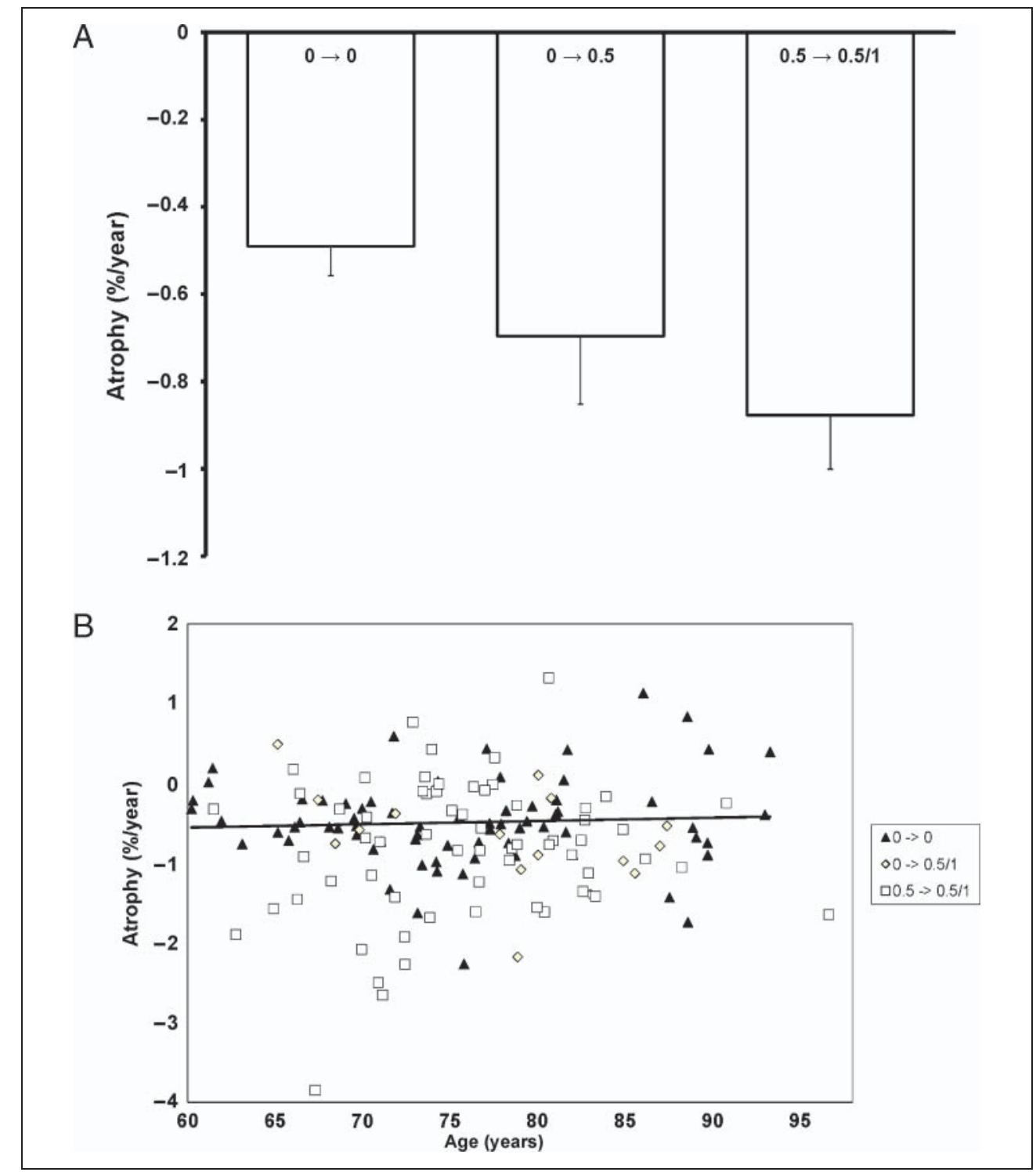


Table 4. Measures Included in the Data Set

\begin{tabular}{ll}
\hline Age & Age at time of image acquisition (years) \\
Sex & Sex (M or F) \\
Education & Years of education \\
SES & $\begin{array}{l}\text { Socioeconomic status as assessed by the Hollingshead Index of Social Position and classified } \\
\text { into categories from } 1 \text { (bighest status) to } 5 \text { (lowest status) (Hollingshead, 1957) }\end{array}$ \\
MMSE & Mini-Mental State Examination score (range is from $0=$ worst to $30=$ best) (Folstein, Folstein, \& McHugh, 1975) \\
CDR & Clinical Dementia Rating $(0=$ no dementia, $0.5=$ very mild AD, $1=$ mild AD, $2=$ moderate AD) (Morris, 1993) \\
ASF & Atlas scaling factor (unitless). Computed scaling factor that transforms native-space brain and \\
& skull to the atlas target (i.e., the determinant of the transform matrix) (Buckner et al., 2004) \\
eTIV & Estimated total intracranial volume (cm $\left.{ }^{3}\right)$ (Buckner et al., 2004) \\
nWBV & Normalized whole-brain volume, expressed as a percent of all voxels in the atlas-masked image that \\
& are labeled as gray or white matter by the automated tissue segmentation process (Fotenos et al., 2005) \\
\hline
\end{tabular}

\section{Obtaining and Using the Data}

OASIS data can be obtained at http://www.oasis-brains.org. Requests for DVD distributions of the data can be submitted at the Web site. For convenience, we also provide the data using the open-source Extensible Neuroimaging Archive Toolkit (Marcus, Olsen, et al., 2007). It provides tools to search, to visualize, and to download the data. Before downloading and requesting data, users are asked to abide by the OASIS Data Usage Agreement.

OASIS data are distributed in GNU zip archive files, which can be uncompressed using freely available software. All images are distributed in NIFTI1 format (http://nifti.nimh. nih.gov), which can be visualized and processed using many common commercial and open source image viewing applications, including Neurolens, ImageJ, Slicer, and MRIcro. For each imaging session, the following image files are included in the distribution: three to four individual scan images; an image in which the individual scans have been aligned and coregistered, an image that has been gain fieldcorrected and registered to the Talairach and Tournoux atlas (T88); a masked T88 image in which the intensity of all nonbrain voxels has been set to zero; and a segmented T88 image in which each voxel has been labeled as gray matter, white matter, or cerebral spinal fluid. Demographic, clinical, and derived imaging measures (Table 4) are available in XML and spreadsheet formats. Additional details of the directory structure, file naming scheme, and image characteristics can be found at http://www.oasis-brains. org/longitudinal_facts.html.

\section{DISCUSSION}

The present data set includes longitudinally acquired T1-weighted MRI data from 150 individuals aged 60 to 96 years, including 64 individuals initially determined to have $\mathrm{AD}$ and 14 who were diagnosed with $\mathrm{AD}$ at a return visit. Repeated within-visit acquisitions are included for each subject allowing extremely high contrast properties after image averaging. The data have been anonymized, carefully screened for image quality, and postprocessed to generate common anatomic measures. The data are available under a liberal usage policy that allows free access and unrestricted usage to all interested parties.

The image files included in this OASIS set differ from the previous OASIS set in two substantive ways meant to improve overall usability. First, the files are in the NiFTI 1 image format, which is now widely supported and preferred for its more explicit specification of voxel ordering. Second, the images have not been altered to remove facial features. This ensures that fiducial markers placed on the left temple are always present in raw scan images. It also removes hard edges that may cause difficulty in some segmentation algorithms.

The specific anatomical measure of nWBV included here illustrates a common approach to analyzing anatomical characteristics of the brain in MRI images, particularly in relation to aging. Unsurprisingly, our findings are in agreement with those described in previous studies using portions of these data (e.g., Fotenos et al., 2005). In particular, nWBV was shown to decline significantly with increasing age and by $\mathrm{AD}$ status as previously reported by Fotenos et al. (2005) and others (Dickerson et al., 2006; Killiany et al., 2000, 2002; Fox \& Freeborough, 1997; Jack et al., 1992, 1997). Similarly, the rate of nWBV decline was significantly greater for the AD group than for the nondemented group.

These findings are representative of the types of analyses that have been reported in the literature and are not intended to be comprehensive or the final word on how to approach these data. Such issues as the interplay between imaging markers, AD, and other diseases (e.g., hypertension, diabetes) remain open avenues for further investigation. Similarly, alternative image processing and statistical methods may yield additional findings. We hope that the description and open release of these data will encourage 
ongoing exploration of the data, leading to improvements in the diagnosis and treatment of $\mathrm{AD}$.

\section{Acknowledgments}

The authors thank the Washington University ADRC and the Conte Center for clinical assistance and participant recruitment; Elizabeth Grant for assistance with data preparation; Susan Larson, Amy Sanders, Laura Williams, Jamie Parker, and Glenn Foster for assistance with MRI data collection; Avi Snyder for development of analytic techniques; and Tim Olsen, Mohana Ramaratnam, Kevin Archie, and Mikhail Milchenko for development of database and Web tools. Anders Dale assisted with the original selection of imaging parameters. The acquisition of this data and the support for data analysis and management were provided by the National Institutes of Health grant nos. P50 AG05681, P01 AG03991, R01 AG021910, P20 MH071616, RR14075, RR 16594, and BIRN002; the Alzheimer's Association; the James S. McDonnell Foundation; the Mental Illness and Neuroscience Discovery Institute; and the Howard Hughes Medical Institute.

Reprint requests should be sent to Daniel S. Marcus, Department of Radiology, Campus Box 8225, Washington University School of Medicine, 4525 Scott Ave., St. Louis, MO 63110, or via e-mail: dmarcus@wustl.edu.

\section{REFERENCES}

Berg, L., McKeel, D. W., Miller, J. P., Storandt, M., Rubin, E. H., Morris, J. C., et al. (1998). Clinicopathologic studies in cognitively healthy aging and Alzheimer disease-Relation of histologic markers to dementia severity, age, sex, and apolipoprotein E genotype. Archives of Neurology, 55, 326-335.

Borroni, B., Premi, E., Di Luca, M., \& Padovani, A. (2007). Combined biomarkers for early Alzheimer disease diagnosis. Current Medicinal Chemistry, 14, 1171-1180.

Buckner, R. L., Head, D., Parker, J., Fotenos, A. F., Marcus, D. S., Morris, J. C., et al. (2004). A unified approach for morphometric and functional data analysis in young, old, and demented adults using automated atlas-based head size normalization: Reliability and validation against manual measurement of total intracranial volume. Neuroimage, 23, 724-738.

Buckner, R. L., Snyder, A. Z., Shannon, B. J., LaRossa, G., Sachs, R., Fotenos, A. F., et al. (2005). Molecular, structural, and functional characterization of Alzheimer's disease: Evidence for a relationship between default activity, amyloid, and memory. Journal of Neuroscience, 25, 7709-7717.

Burns, J. M., Church, J. A., Johnson, D. K., Xiong, C., Marcus, D., Fotenos, A. F., et al. (2005). White matter lesions are prevalent but differentially related with cognition in aging and early Alzheimer disease. Archives of Neurology, 62, 1870-1876.

Chan, D., Fox, N. C., Jenkins, R., Scahill, R. I., Crum, W. R., \& Rossor, M. N. (2001). Rates of global and regional cerebral atrophy in $\mathrm{AD}$ and frontotemporal dementia. Neurology, 57, 1759-1763.

Chong, M. S., Lim, W. S., \& Sahadevan, S. (2006). Biomarkers in preclinical Alzheimer's disease. Current Opinion in Investigational Drugs, 7, 600-607.

Dickerson, B. C., Bakkour, A., Salat, D. H., Feczko, E., Pacheco, J., Greve, D. N., et al. (2008). The cortical signature of Alzheimer's disease: Regionally-specific cortical thinning relates to symptom severity in very mild to mild $\mathrm{AD}$ dementia and is detectable in asymptomatic amyloid-positive individuals. Cerebral Cortex, 19, 497-510.

Dickerson, B. C., Feczko, E., Augustinack, J. C., Pacheco, J., Morris, J. C., Fischl, B., et al. (2009). Differential effects of aging and Alzheimer's disease on medial temporal lobe cortical thickness and surface area. Neurobiology of Aging, 30, 432-440.

Dickerson, B. C., Goncharova, I., Sullivan, M. P., Forchetti, C., Wilson, R. S., Bennett, D. A., et al. (2006). Longitudinal course and neuropathologic outcomes in original vs revised MCI and in pre-MCI. Neurology, 67, 467-473.

Folstein, M. F., Folstein, S. E., \& McHugh, P. R. (1975). "Mini-mental state". A practical method for grading the cognitive state of patients for the clinician. Journal of Psychiatric Research, 12, 189-198.

Fotenos, A. F., Mintun, M. A., Snyder, A. Z., Morris, M. D., \& Buckner, R. L. (2008). Brain volume decline in aging: Evidence for a relation between socioeconomic status, preclinical Alzheimer's disease, and reserve. Archives of Neurology, 65, 113-120.

Fotenos, A. F., Snyder, A. Z., Girton, L. E., Morris, J. C., \& Buckner, R. L. (2005). Normative estimates of cross-sectional and longitudinal brain volume decline in aging and $\mathrm{AD}$. Neurology, 64, 1032-1039.

Fox, N. C., \& Freeborough, P. A. (1997). Brain atrophy progression measured from registered serial MRI Validation and application to Alzheimer's disease. Journal of Magnetic Resonance Imaging, 7, 1069-1075.

He, Y., Chen, Z., \& Evans, A. (2008). Structural insights into aberrant topological patterns of large-scale cortical networks in Alzheimer's disease. Journal of Neuroscience, 28, 4756-4766.

Head, D., Snyder, A. Z., Girton, L. E., Morris, J. C., \& Buckner, R. L. (2005). Frontal-hippocampal double dissociation between normal aging and Alzheimer's disease. Cerebral Cortex, $15,732-739$.

Hollingshead, A. (1957). Two factor index of social position. New Haven, CT: Yale University Press.

Jack, C. R., Jr., Petersen, R. C., O’Brien, P. C., \& Tangalos, E. G. (1992). MR-based hippocampal volumetry in the diagnosis of Alzheimer's disease. Neurology, 62, 183-188.

Jack, C. R., Jr., Petersen, R. C., Grundman, M., Jin, S., Gamst, A., Ward, C. P., Sencakova, D., Doody, R. S., Thal, L. J., \& Members of the Alzheimer's Cooperative Study. (2008). Longitudinal MRI findings from the vitamin $\mathrm{E}$ and donepezil treatment study for MCI. Neurobiology of Aging, 29, 1285-1295.

Jack, C. R., Jr., Petersen, R. C., Xu, Y. C., Waring, S. C., O’Brien, P. C., Tangalos, E. G., et al. (1997). Medial temporal atrophy on MRI in normal aging and very mild Alzheimer's disease. Neurology, 49, 786-794.

Jack, C. R., Jr., Shiung, M. M., Gunter, J. L., O’Brien, P. C., Weigand, S. D., Knopman, D. S., et al. (2004). Comparison of different MRI brain atrophy rate measures with clinical disease progression in AD. Neurology, 62, 591-600.

Killiany, R. J., Gomez-Isla, T., Moss, M., Kikinis, R., Sandor, T., Jolesz, F., et al. (2000). Use of structural magnetic resonance imaging to predict who will get Alzheimer's disease. Annals of Neurology, 47, 430-439.

Killiany, R. J., Hyman, B. T., Gomez-Isla, T., Moss, M. B., Kikinis, R., Jolesz, F., et al. (2002). MRI measures of entorhinal cortex vs hippocampus in preclinical AD. Neurology, 58, 1188-1196.

Liu, R., Lemieux, L., Bell, G. S., Sisodiya, S. M., Shorvon, S. D., Sander, J. W., et al. (2003). A longitudinal study of brain morphometrics using quantitative magnetic resonance imaging and difference image analysis. Neuroimage, 20, 22-33.

Marcus, D. S., Olsen, T. R., Ramaratnam, M., \& Buckner, R. L. (2007). The Extensible Neuroimaging Archive Toolkit: 
An informatics platform for managing, exploring, and sharing neuroimaging data. Neuroinformatics, 5, 11-34.

Marcus, D. S., Wang, T. H., Parker, J., Csernansky, J. G., Morris, J. C., \& Buckner, R. L. (2007). Open Access Series of Imaging Studies (OASIS): Cross-sectional MRI data in young, middle aged, nondemented, and demented older adults. Journal of Cognitive Neuroscience, 19, 1498-1507.

Morris, J. C. (1993). The Clinical Dementia Rating (CDR): Current version and scoring rules. Neurology, 43, 2412-2414.

Morris, J. C., Storandt, M., Miller, J. P., McKeel, D. W., Price, J. L., Rubin, E. H., et al. (2001). Mild cognitive impairment represents early-stage Alzheimer disease. Archives of Neurology, 58, 397-405.

Mueller, S. G., Weiner, M. W., Thal, L. J., Petersen, R. C., Jack, C. R., Jagust, W., et al. (2005). Ways toward an early diagnosis in Alzheimer's disease: The Alzheimer's Disease Neuroimaging Initiative (ADNI). Alzheimer's \& Dementia, 1, 55-60.

Raz, N., Lindenberger, U., Rodrigue, K. M., Kennedy, K. M., Head, D., Williamson, A., et al. (2005). Regional brain changes in aging healthy adults: General trends, individual differences and modifiers. Cerebral Cortex, 15, 1676-1689.

Resnick, S. M., Pham, D. L., Kraut, M. A., Zonderman, A. B., \& Davatzikos, C. (2003). Longitudinal magnetic resonance imaging studies of older adults: A shrinking brain. Journal of Neuroscience, 23, 3295-3301.

Salat, D. H., Buckner, R. L., Snyder, A. Z., Greve, D. N., Desikan, R. S. R., Busa, E., et al. (2004). Thinning of the cerebral cortex in aging. Cerebral Cortex, 14, 721-730.
Salat, D. H., Greve, D. N., Pacheco, J. L., Quinn, B. T., Helmer, K. G., Buckner, R. L., et al. (2009). Regional white matter volume differences in nondemented aging and Alzheimer's disease. Neuroimage, 15, 1247-1258.

Scahill, R. I., Schott, J. M., Stevens, J. M., Rossor, M. N., \& Fox, N. C. (2002). Mapping the evolution of regional atrophy in Alzheimer's disease: Unbiased analysis of fluid-registered serial MRI. Proceedings of the National Academy of Sciences, U.S.A., 99, 4703-4707.

Storandt, M., Grant, E. A., Miller, J. P., \& Morris, J. C. (2006). Longitudinal course and neuropathologic outcomes in original vs revised MCI and in pre-MCI. Neurology, 67, 467-473.

Talairach, J., \& Tournoux, P. (1988). Co-planar stereotaxic atlas of the buman brain: An approach to medical cerebral imaging. New York: Thieme.

Thompson, P. M., Hayashi, K. M., de Zubicaray, G., Janke, A. L., Rose, S. E., Semple, J., et al. (2003). Dynamics of gray matter loss in Alzheimer's disease. Journal of Neuroscience, 23, 994-1005.

Wang, D. M., \& Doddrell, D. M. (2002). MR image-based measurement of rates of change in volumes of brain structures. Part 1: Method and validation. Magnetic Resonance Imaging, 20, 27-40.

Wang, L., Swank, J. S., Glick, I. E., Gado, M. H., Miller, M. I., Morris, J. C., et al. (2003). Changes in hippocampal volume and shape across time distinguish dementia of then Alzheimer type from healthy aging. Neuroimage, 20, 667-682. 